\title{
Mindfulness per l'età evolutiva
}

di Maria Beatrice Toro*

La mindfulness, pratica messa a punto da Jon Kabat Zinn negli anni '90, viene sinteticamente definita come la consapevolezza che emerge quando si direziona l'attenzione, intenzionalmente, a ciò che accade nel presente, secondo una modalità non giudicante.

Attraverso tale metodo, negli ultimi tre decenni, un numero imponente di persone ha migliorato il proprio stato di benessere e ha imparato a mettersi al riparo dallo stress, stabilizzando la mente e il cuore attraverso un'attitudine particolare verso la vita, ovvero un'apertura serena, accettante e grata al momento presente (Kabat Zinn, 1994).

Le pratiche e gli esercizi, opportunamente adattati per le diverse età, possono risultare utili anche per i bambini, funzionando come una guida rispettosa che li porta, delicatamente, a scoprire la loro mente e il loro cuore, sottraendoli all'incessante bisogno di esperienze eccitanti in cui a volte sembrano smarrirsi e dalle quali, poi, possono diventare dipendenti.

I vantaggi della formazione alla consapevolezza per i bambini (in questo articolo ci si riferisce a un'età compresa tra i cinque e i dodici anni) sono molti: meno ansia, maggiore attenzione, migliore intelligen\section{za emotiva.}

Le competenze sociali ed emotive che essa aiuta a far fiorire, li porteranno a essere più in grado di capirsi, ascoltarsi, comunicare, essere più gentili con se stessi e con gli altri.

I bambini possono apprendere tutto ciò con una naturalezza sorprendente. Saranno loro, ben presto, a mostrare a noi la bellezza semplice del-

* Psicologa, psicoterapeuta, Direttore didattico della SCInt - Scuola di Specializzazione in Psicoterapia Cognitivo Interpersonale (Aut. Miur. 2008), Direttore Secondo Centro di Terapia Cognitivo Interpersonale, Roma. 
l'esplorare la vita con uno sguardo privo di preconcetti, alla scoperta delle qualità uniche e irripetibili di ogni istante.

\section{Direzionare l'attenzione}

Come efficacemente riassunto da Toro e Serafinelli (2015), il processo fondamentale descritto da Jon Kabat Zinn presenta le tre caratteristiche seguenti: l'intenzionalità, l'orientamento al presente, la modalità non giudicante.

La prima di queste qualità ha a che fare con la cura e l'impegno intenzionale che è necessario investire sul processo attenzionale, per riuscire a osservare i meccanismi mentali automatici; la seconda qualità dell'attenzione mindful consiste nell'orientamento al presente: l'unico momento in cui possiamo stabilirci, osservare, agire. Esso rappresenta, in un certo senso, l'unico momento che esiste, ma, paradossalmente, è quello cui ci dedichiamo meno, tornando con la mente al passato, $\mathrm{o}$ anticipando ciò che accadrà nel futuro. Spesso incontriamo, altresì, una resistenza nello stare con il presente, magari perché non ci soddisfa o non corrisponde al presente che vorremmo.

Per questo motivo la pratica di mindfulness viene effettuata secondo una terza fondamentale direttrice, quella del non giudizio. È proprio tale attitudine non giudicante che ci consente di abitare la vita così come è ora, senza aspettare che sia come l'abbiamo progettata (Toro e Serafinelli, 2015).

L'utilizzo di tale metodologia con bambini che, come sappiamo, tendono oggi a fare molte cose tutte insieme e a incontrarsi virtualmente più che di persona, appare uno dei modi che abbiamo per trasmettere alle nuove generazioni tutta l'importanza dell'essere presenti a se stessi e dell'essere insieme, del fare una cosa per volta, del conoscere l'altro senza pregiudizi. Come è naturale, ciò potrà essere insegnato da adulti che a loro volta siano in grado di dare ai bambini tutta l'attenzione che serve, senza fretta e senza distrazioni. In parole semplici, potemmo dire che se, come adulti, sappiamo liberarci noi per primi dallo stress, prendendoci il tempo che ci occorre per essere pienamente presenti a noi stessi, poi, possiamo aiutare i bambini e i ragazzi ad appropriarsi serenamente del loro tempo, prendendosi il gusto di vivere fino in fondo la bellezza dell'infanzia.

\section{Evidenze scientifiche}

Sebbene si tenda ad associare il concetto di stress alla vita adulta, tale stato psicofisiologico si può riscontrare anche in età infantile. Purtroppo, tuttavia, il fenomeno è rimasto finora piuttosto misconosciuto, cosicché 
molti adulti, sopravvalutando le capacità del bambino di fare fronte alle molte sfide di una vita densa di impegni e sovraccarica di stimoli, chiedono al bambino prestazioni e autonomie che superano le sue possibilità.

Lo stress interferisce negativamente sulla percezione che il bambino ha di sé come persona competente e adeguata, deprimendo la naturale tendenza alla socializzazione con i pari, ma anche la voglia di apprendere a scuola, il piacere di fare sport. Stress e ansia sono associabili a difficoltà di attenzione (Ehrenreich e Gross, 2002), distorsioni cognitive, labilità emotiva (Mineka e Gilboa, 1998), ipervigilanza fisiologica.

Come scrivono Montano e Villani in un recente testo che presenta un programma italiano di mindfulness per bambini, Il fiore dentro,

sono molto diffusi tra i bambini l'ansia cronica, i disturbi alimentari, l'ADHD e l'autolesionismo. (...) È evidente come i bambini che imparano precocemente a condurre uno stile di vita passivo e sedentario, senza volizione e con una forte inclinazione all'evitamento, seguiteranno a indugiare in uno stato simile per il resto della vita, con gravi conseguenze sul piano della salute mentale e fisica: obesità, diabete, incapacità di tollerare lo stress, inclinazione alla violenza e all'uso di stupefacenti sono solamente alcuni tra gli esiti più lampanti di un comportamento malsano perpetuato e fuori controllo (Montano e Villani, 2016).

L'attenzione, in particolare, è stata costantemente associata ad apprendimento e rendimento un po' in tutte le discipline in cui il bambino normalmente si cimenta (Duncan et al., 2007). Una recente review riguardante l'applicazione di protocolli mindfulness con bambini, curata da Mark Greenberg e Alexis Harris (2011), indica l'efficacia di queste pratiche nello sviluppo della resilienza in bambini che non presentano particolari problemi emotivi o comportamentali e ne rileva una buona utilizzabilità anche $\mathrm{e}$ nel trattamento di disordini psicologici.

Questa review suggerisce che la meditazione può essere associata a esiti positivi, nonostante i dati a disposizione siano relativi a esperienze recenti e ci sia, indubbiamente, ancora molto da approfondire.

Uno studio davvero molto interessante, poi, collega la qualità e la durata dell'attenzione del bambino alla presenza incoraggiante e sicura di un adulto. L'esperimento è stato ideato da due psicologi dell'Indiana University e pubblicato sulla rivista Current Psychology. Mostra un collegamento diretto fra l'attenzione che il genitore dedica effettivamente al bambino durante un gioco condiviso e il tempo in cui il bambino riesce a rimanere attento, focalizzandosi assieme al genitore su quello stesso oggetto di gioco. In particolare si è visto che il vagare dello sguardo lontano dal gioco durante la sessione sperimentale, influisce negativamente sulla curva temporale dell'attenzione. Ora, la capacità dei bambini di sostenere l'attenzione rappresenta un indicatore importantissimo 
per il processo di crescita psicologica, poiché favorisce l'acquisizione di un linguaggio articolato, la capacità di risoluzione di problemi e altre pietre miliari cognitive. Chen $\mathrm{Yu}$, ideatore dello studio e Linda Smith, hanno dimostrato una cosa importantissima e inedita: la genesi della facoltà attentiva è profondamente incastonata nel contesto dell'interazione sociale.

Lo stato mentale di presenza vigile e concentrata dell'adulto influenza significativamente il bambino, più che la quantità di stimoli disponibili. Grazie a un macchinario montato su un casco gli studiosi hanno monitorato genitore e figlio mentre giocavano. I bimbi con livelli di attenzione ottimale, erano quelli appartenenti alle coppie più funzionali, caratterizzate da una serie di caratteristiche interattive: il genitore cominciava la sessione restando in attesa paziente che il bambino iniziasse col suo gioco preferito, lasciandogli libertà di scelta, assumeva un atteggiamento cognitivo di reale concentrazione sull'attività da lui proposta, nominava l'oggetto scelto dal bimbo e parlava delle sue caratteristiche (ad es. «Ecco l'orsacchiotto! Come è morbido!»); evitava, inoltre, di guardare altrove o distrarsi e incoraggiava il figlio a proseguire il gioco insieme.

Risultati peggiori erano invece ottenuti da genitori troppo invadenti, che gestivano il gioco dirigendolo e, ancor più, da quelli che si mostravano passivi, disinteressati. Ovviamente possiamo ipotizzare che tutto ciò abbia una certa rilevanza non solo dal punto di vista cognitivo, ma anche per quanto concerne la sfera affettiva e la soddisfazione del bambino.

\section{Adattamenti}

I protocolli di mindfulness per gli adulti utilizzano, fondamentalmente, tre modalità di meditazione di consapevolezza (body scan, pratica sul respiro, mindful yoga), mentre, per i bambini, viene prevista una maggiore varietà di esercizi sensoriali (visivi, uditivi, tattili, olfattivi, gustativi, cinestesici), per rendere il metodo più concreto e semplice. Le interazioni di gruppo, per gli adulti, consistono in larga parte in descrizioni e scambi verbali, mentre le interazioni di gruppo per i bambini possono includere giochi, e, in generale, è opportuno proporre più attività in movimento: gli esercizi di consapevolezza sono più brevi e più vari nel corso di ciascuna sessione, in cui si alternano osservazioni multisensoriali, brevi meditazioni sul respiro, body scan, movimenti, visualizzazioni, disegno, scrittura. Per il bambino, infatti, rispetto al modo di fare esperienza adulto, c'è un minore investimento logico verbale, prevalendo una modalità concreta. Per quanto riguarda il tema dell'ansia, in particolare, vi sono importanti specificità; i bambini hanno solitamente paure più specifiche (della scuola, dell'interrogazione, della morte di una persona) e 
più sintomi somatici. Gli adulti ansiosi riferiscono preoccupazioni più generali, come sentirsi incapaci, o sopraffatti. I programmi psicoeducativi che utilizzano una varietà di approcci multisensoriali si sono dimostrati più efficaci (Semple et al., 2009). Nei protocolli per bambini è opportuno coinvolgere anche i genitori, invitandoli a impegnarsi con la partecipazione a una o due sessioni di mindfulness (cf. anche Montano e Villani, 2016). Essi possono essere incoraggiati anche a partecipare alla pratica a casa e, soprattutto, a impegnarsi ad adottare uno "stile" genitoriale consapevole.

\section{Trattare l'ansia e aumentare la resilienza con la mindfulness}

L'ansia rappresenta uno dei problemi infantili più diffusi ed è uno degli effetti dello stress sul bambino. Come psicoterapeuti, sappiamo che il trattamento cognitivo comportamentale risulta efficace nella maggior parte dei casi, anche dopo un follow up di tre anni dal trattamento. Possiamo aumentare i benefici dei trattamenti includendo la pratica di mindfulness tra gli strumenti a disposizione del clinico?

Per comprenderlo, ci dobbiamo anzitutto chiedere in quali processi può intervenire e quali componenti del trattamento possa rafforzare. Stando alle evidenze tratte da esperienze terapeutiche rivolte ad adulti, possiamo ipotizzare che il punto cruciale che rende la mindfulness efficace è che consente, più che di cambiare i contenuti mentali, di cambiare il rapporto che il soggetto intrattiene con tali contenuti. Tutte le pratiche, infatti, enfatizzano l'osservazione dell'esperienza interna (Semple, Reid e Miller, 2005); un'osservazione "spassionata", nudamente imparziale, non valutativa e non reattiva. Essa aiuta a prendere contatto con l'esperienza interna così come si presenta momento dopo momento, discriminando tale esperienza dalla sua eventuale relazione con l'esterno. Un altro punto qualificante dei protocolli mindfulness based è che essi possono essere proposti anche a gruppi piuttosto numerosi con evidente vantaggio a livello di efficienza. Per i bambini, il fatto di apprendere uno strumento di autoregolazione ha un'altra conseguenza importante: assumere un ruolo attivo è indirettamente benefico per il senso di auto efficacia percepita (Semple, Reid e Miller, 2005).

La terapia cognitiva basata sulla Mindfulness per bambini (MBCT-C) è stata "manualizzata" sulla base dei risultati nella riduzione dell'ansia ottenuti da Semple. È un protocollo sviluppato per bambini di 9-13 anni, per aumentare la resilienza sia a livello emozionale (gestire ansia e stress) che sociale (assumere comportamenti adeguati nelle interazioni con i pari). Semple et al. (2009) hanno testato, in particolare, tre ipotesi in uno studio che ha coinvolto 25 bambini: 
a) l'MBCT-C riduce significativamente i problemi di attenzione;

b) riduce l'ansia;

c) migliora i problemi di comportamento.

Le misure utilizzate hanno incluso la Child Behavior Checklist, lo StateTraitAnxiety Inventory per i bambini, nonché la scala multidimensionale di misura dell'ansia MASC. Lo studio ha mostrato l'esistenza di una forte correlazione tra i problemi di attenzione e i problemi di comportamento (i problemi di attenzione spiegano il $46 \%$ della varianza dei cambiamenti nel comportamento) e un miglioramento, dopo l'MBCT-C, in ambedue le variabili. Riduzioni significative dei sintomi ansiosi sono stati riscontrati, relativamente a quei bambini che hanno raggiunto indici di ansia clinicamente elevati al pretest. I risultati mostrano che la mindfulness è uno strumento promettente per migliorare attenzione, stato emozionale, comportamento sociale.

\section{Conclusioni e... un esercizio!}

Come abbiamo scritto, anche il bambino si preoccupa (per la scuola, per i voti, persino per la salute dei genitori); immagina problemi che non esistono cercando di risolverli prima che si siano effettivamente presentati. Il suo film mentale è molto concreto, si basa su qualche fatto passato e prevede eventi negativi, o si concentra su ciò che non c'è, vivendo stati di frustrazione e rabbia, come nel caso in cui gli venga opposto un rifiuto allorché richieda, ad esempio, un gioco che il genitore non ritiene adatto.

Per renderli "più felici", possiamo aiutarli - più che acquistando qualcosa o proponendo un'attività nuova - insegnando loro che tutto ciò di cui hanno bisogno per essere felici c'è già, ma non lo focalizzano perché sono sopraffatti da paure e rimpianti per bisogni insoddisfatti, che, magari, sono solo capricci. Spieghiamo al bambino che dentro ognuno di noi c'è un'oasi di felicità e sicurezza: per entrarci bisogna lasciare fuori oggetti, giocattoli, ma anche preoccupazioni e pensieri. Prima della pratica facciamogli scrivere in un bigliettino se c'è un pensiero che gli dà fastidio in questo momento e poi lo prendiamo in consegna, per lasciare che il bambino sia libero di fare un giro nella sua oasi personale senza questo pensiero. Facciamo salutare il bigliettino dicendo: «ci vediamo dopo!».

Questo serve a far vedere al bambino che anche se si rilassa per un certo tempo, dopo sarà possibile, se lo vuole, riprendersi le sue preoccupazioni. Intanto, però, avrà "liberato" un pezzettino del suo tempo da un peso. Più si fiderà e più sarà in grado, quando il pensiero molesto si presenta in qualunque contesto, di dirgli: «Ok, ti ho visto, ora ho da fare, ci vediamo dopo!». 
Troviamo un posto tranquillo in cui poter restare per un po' e poi invitiamo il bambino a stare seduto rilassato. Posizioniamo quattro disegni che abbiamo fatto fare in precedenza, che rappresentano: il sole, il cielo, un fiore, l'oasi.

Facciamo rivolgere l'attenzione al loro respiro, dicendo di ascoltare le sensazioni dell'aria che entra ed esce dal corpo. Si possono far poggiare le mani sull'ombelico per percepire il dolce movimento che alza e abbassa la pancia: questo li aiuterà a mantenere la concentrazione.

Chiediamo, di ripetere le parole "in" e "es", per tre inspirazioni ed espirazioni.

Poi immaginiamo il sole, bello, giallo, tiepido, sorridente. Inspirando, faccio entrare il sole. Espirando, mi sento luminoso.

Sole, luminoso (3 respiri)

Poi presentiamo il cielo azzurro e pulito, nitido, senza nuvole. Inspirando mi vedo come il cielo, espirando, mi sento limpido.

Cielo, limpido (3 respiri)

Poi immaginiamo di intravedere, sotto il cielo, in mezzo a della terra bianca, una rosa del deserto. Inspirando mi vedo come un fiore, colorato, espirando, mi sento meraviglioso.

\section{Fiore, meraviglioso (3 respiri)}

Adesso scorgiamo un'oasi e capiamo che il fiore è cresciuto proprio in riva a un'oasi di acqua fresca e chiara, dove tutto è meraviglioso e sicuro. Dovunque mi giro, vedo cose belle, anche dietro di me, dove non posso vedere, so che tutto è in armonia e in pace. L'oasi è felice che io sia lì. Posso immaginare alberi e animali che si rilassano nell'acqua chiara. Il fiore, gli alberi, gli animali, sono felici che io sia lì. Inspirando, mi vedo come in questo scenario, espirando, mi sento libero.

\section{Spazio sicuro, spazio libero (3 respiri)}

\section{Sommario}

Attraverso i protocolli mindfulness un numero imponente di persone ha migliorato il proprio stato di benessere e ha imparato a mettersi al riparo dallo stress, stabilizzando la mente e il cuore attraverso un'attitudine particolare verso la vita, ovvero un'apertura serena, accettante e grata al momento presente (Kabat Zinn, 1994). Nell'articolo si presenta la letteratura riguardo a interventi mindfulness per i bambini. Le pratiche e gli esercizi, opportunamente adattati per le diverse età, possono risultare utili per diminuire ansia e stress, migliorare l'attenzione e la qualità delle interazioni sociali.

Parole chiave: mindfulness, mindfulness per i bambini, esercizi di mindfulness, attenzione, attenzione condivisa. 


\section{Abstract. Mindfulness for children}

Through mindfulness programs an impressive number of people have improved their well-being and learnt to take shelter from stress, stabilizing mind and heart through a particular attitude towards life: an accepting and grateful openness to the present moment (Kabat Zinn, 1994). The article presents scientidic literature about mindfulness interventions for children. Practices and exercises, suitably adapted for different ages, may help children to reduce anxiety and stress, to improve attention and to enhance quality of social interactions.

Key words: mindfulness, children, mindfulness exercises, attention, shared attention.

\section{Riferimenti bibliografici}

Duncan G.J., Dowsett C.J., Claessens A., Magnuson K., Huston A.C., Klebanov P., et al. (2007), "School readiness and later achievement", Developmental Psychology, 43: 1428-1446. DOI: 10.1037/0012-1649.43.6.1428

Ehrenreich J.T. e Gross A.M. (2002), "Biased attentional behavior in childhood anxiety a review of theory and current empirical investigation", Clinical Psychology Review, 22: 991-1008.

Greenberg M.T. e Harris A.R. (2012), "Nurturing mindfulness in children and youth: Current state of research", Child Development Perspectives, 6, 2: 161-166. DOI: 10.1111/j.1750-8606.2011.00215.x

Mineka S. e Gilboa E. (1998), Cognitive biases in anxiety and depression, in William J., Flack F. e Laird J.D. (eds.), Emotions in psychopathology: Theory and research (216-228), New York: Oxford University Press.

Montano A. e Villani S. (2016), Programma mindfulness «il fiore dentro». Per insegnare ai bambini a gestire lo stress ed essere più felici, Eclipsi, Firenze.

Semple R., Reid E. e Miller L. (2005), "Treating Anxiety with Mindfulness: An Open Trial of Mindfulness Training for Anxious Children", Journal of Cognitive Psychotherapy, 19, 4: 379-392 (14). DOI: 10.1891/jcop.2005.19.4.379

Toro M.B. e Serafinelli S. (2015), Mindfulness Insieme. Coltivare la consapevolezza con se stessi, in coppia e sul lavoro, Milano: Franco Angeli.

www.mindfulnessinterpersonale.com 